OPEN ACCESS

Edited by:

Carmine De Angelis,

University of Naples Federico II, Italy

Reviewed by:

Luca Gelsomino,

University of Calabria, Italy Alessandro Igor Cavalcanti Leal, Johns Hopkins Medicine, United States

*Correspondence Tomás Reinert tomasreinert@hotmail.com

Specialty section: This article was submitted to Women's Cancer,

a section of the journal

Frontiers in Oncology

Received: 10 December 2019 Accepted: 27 February 2020 Published: 03 April 2020

Citation:

Reinert T, Ramalho $S$, de Vasconcelos VCA, Silva $L R$, da Silva AER, de Andrade CA Kraft MBdPL, Coelho GP, Mandelli J, Binotto M, Cabello C, Paiva Silva GRd,

Bines J, Barrios $\mathrm{CH}$, Ellis $\mathrm{MJ}$ and Graudenz MS (2020) ESR1 Mutations

Are Not a Common Mechanism of Endocrine Resistance in Patients With Estrogen Receptor-Positive Breast Cancer Treated With Neoadjuvant Aromatase Inhibitor Therapy. Front. Oncol. 10:342 doi: 10.3389/fonc 2020.0034

\section{ESR1 Mutations Are Not a Common Mechanism of Endocrine Resistance in Patients With Estrogen Receptor-Positive Breast Cancer Treated With Neoadjuvant Aromatase Inhibitor Therapy}

Tomás Reinert ${ }^{1,2,3 *}$, Susana Ramalho ${ }^{4}$, Vivian Castro Antunes de Vasconcelos ${ }^{4}$, Leonardo Roberto Silva ${ }^{4}$, Ana Elisa Ribeiro da Silva ${ }^{4}$, Camila Annicchino de Andrade ${ }^{4}$, Maria Beatriz de Paula Leite Kraft ${ }^{4}$, Guilherme Portela Coelho ${ }^{5}$, Jovana Mandelli ${ }^{5}$, Monique Binotto ${ }^{2}$, Cesar Cabello ${ }^{4}$, Geisilene Russano de Paiva Silva ${ }^{6}$, José Bines ${ }^{7}$, Carlos H. Barrios ${ }^{3}$, Matthew J. Ellis ${ }^{8}$ and Marcia Silveira Graudenz ${ }^{1}$

${ }^{1}$ Postgraduate Program in Medical Sciences, Universidade Federal Do Rio Grande Do Sul (UFRGS), Porto Alegre, Brazil, ${ }^{2}$ Centro de Pesquisa da Serra Gaucha (CEPESG), Caxias Do Sul, Brazil, ${ }^{3}$ Latin American Cooperative Oncology Group (LACOG), Porto Alegre, Brazil, ${ }^{4}$ Department of Obstetrics and Gynecology, Faculty of Medical Sciences, State University of Campinas (UNICAMP), Campinas, Brazil, ${ }^{5}$ Diagnose Patologia e Biologia Molecular, Caxias Do Sul, Brazil, ${ }^{6}$ Laboratory of Molecular and Investigative Pathology - LAPE, Faculty of Medical Sciences, State University of Campinas (UNICAMP), Campinas, Brazil, ${ }^{7}$ Instituto Nacional Do Câncer (INCA - HCIII), Rio de Janeiro, Brazil, ${ }^{8}$ Lester and Sue Smith Breast Cancer Center, Baylor College of Medicine, Houston, TX, United States

Introduction: Mutations in the ESR1 gene (ESR1m) are important mechanisms of resistance to endocrine therapy in estrogen receptor-positive $(E R+)$ metastatic breast cancer and have been studied as a potential therapeutic target, as well as a predictive and prognostic biomarker. Nonetheless, the role of ESR1m as a possible mechanism of primary endocrine resistance, as well as whether it also occurs in tumors that are resistant to ET administered in early-stage disease as (neo)adjuvant, has not been adequately studied. In this study, we evaluated the prevalence of ESR1m in tumor samples from patients with $\mathrm{ER}+$ breast cancer resistant to neoadjuvant aromatase inhibitor therapy.

Methods: We followed a prospective cohort of patients with ER+ HER2- stages II and III breast cancer treated with neoadjuvant endocrine therapy (NET). Tumor samples from patients with a pattern of primary endocrine resistance [defined as a Preoperative Endocrine Prognostic Index (PEPI) score of $\geq 4$ ] were identified and analyzed for the presence of ESR1m.

Results: One hundred twenty-seven patients were included in the cohort, of which 100 (79\%) had completed NET and underwent surgery. Among these patients, the PEPI score ranged from 0 to 3 in 70\% (70/100), whereas 30\% (30/100) had a PEPI score of 4 or more. Twenty-three of these patients were included in the analysis. ESR1 mutations were not identified in any of the 23 patients with early-stage ER+ breast cancer resistant to NET. 
Discussion: Growing evidence supports the notion that there are different mechanisms for primary and secondary endocrine resistance. Our study suggests that ESR1 mutations do not evolve rapidly and do not represent a common mechanism of primary endocrine resistance in the neoadjuvant setting. Therefore, ESR $1 \mathrm{~m}$ should be considered a mechanism of acquired endocrine resistance in the context of advanced disease. Further research should be conducted to identify factors associated with intrinsic resistance to ET.

\section{Keywords: breast cancer, endocrine therapy, ESR1, ESR1 mutations, neoadjuvant, aromatase inhibitors}

\section{INTRODUCTION}

Estrogen receptor-positive $(\mathrm{ER}+)$ breast cancer is the most prevalent breast cancer subtype. Endocrine therapy (ET) remains the mainstay of treatment in all stages of the disease (1). Nevertheless, endocrine resistance associated with disease progression remains an important challenge $(2,3)$. Mutations of the ESR1 gene, which encodes the ER protein, have been increasingly identified as one of the most important mechanisms of endocrine resistance (4).

Breast tumors are known to undergo genomic evolution, and ESR1 mutations (ESR1m) have been identified in 9-40\% of patients with metastatic ER+ breast cancer resistant to aromatase inhibitors (AIs) (3-7). In Brazilian patients with visceral metastasis of ER+ HER2-negative breast cancer, our group reported the presence of ESR $1 \mathrm{~m}$ in $25 \%$ of the cases (8). In the metastatic setting, the presence of ESR $1 \mathrm{~m}$ is a biomarker of a worse prognosis. The function of ESR $1 \mathrm{~m}$ as a potential therapeutic target, as well as a predictive and prognostic biomarker, is being studied (9). However, the role of ESR 1m as a possible mechanism of ET resistance in the early-stage disease is unclear. Regardless of the extensive research that is being conducted in this field, several questions remain unanswered about ESR1m, such as whether it is associated with endocrine resistance to AIs used with a curative intent treatment in the (neo)adjuvant setting.

Neoadjuvant endocrine therapy (NET) is a therapeutic approach that is being increasingly explored, not only to allow less extensive surgery but also as a scientific tool (10). The Preoperative Endocrine Prognostic Index (PEPI) is a surrogate of endocrine sensitivity and identifies a subgroup of patients with primary resistance to $\operatorname{NET}(11,12)$.

The objective of this study was to evaluate the potential role of ESR $1 \mathrm{~m}$ as a mechanism of resistance in postmenopausal patients with breast cancer treated with NET with a high PEPI score as a surrogate for primarily endocrine-resistant biology.

\section{METHODS}

We conducted a prospective cohort of patients with breast cancer treated with NET in two institutions. The population included postmenopausal women presenting with stages II and III ER+ Her2-negative breast cancer. Protocols of diagnosis, therapies, and follow-up of patients were standardized and based on major international guidelines. These procedures are not part of the study.

Eligible patients were treated with NET with anastrozole for a recommended period of at least 3 months, followed by surgery. Pathological and immunohistochemistry (IHC) protocols were standardized and followed American Society of Clinical Oncology/College of American Pathologists guidelines and the international consensus of pathologic assessment of the breast and axilla after preoperative therapy (13). For all patients who underwent surgery, the PEPI score was calculated (described in Table 1). All IHC assessments were reviewed by two pathologists. Tumor samples from patients with a pattern of primary endocrine-resistant tumors (defined as a PEPI score of $\geq 4$ ) were selected and analyzed for the presence of ESR $1 \mathrm{~m}$.

ESR 1m were evaluated in formalin-fixed paraffin-embedded breast cancer tissue using real-time quantitative polymerase

TABLE 1 | The preoperative endocrine prognostic index (PEPI) score following 4-6 months of neoadjuvant Al or other endocrine therapy provides another strategy to identify endocrine-sensitive vs. endocrine-resistant tumors in the early-stage setting.

\begin{tabular}{lcc}
\hline Surgical factors & RFS HR & PEPI sco \\
\hline TUMOR SIZE & - & 0 \\
T1/T2 & 2.8 & 3 \\
T3/T4 & & \\
NODE STATUS & - & 0 \\
Negative & 3.2 & 3 \\
Positive & & \\
Ki67 LEVEL & - & 0 \\
0-2.7\% & 1.3 & 1 \\
$>$ 2.7-7.3\% & 1.7 & 1 \\
$>7.3-19.7 \%$ & 2.2 & 2 \\
$>19.7-53.1 \%$ & 2.9 & 3 \\
$>53.1 \%$ & & 3 \\
ER STATUS & 2.8 & 0 \\
Negative & 0 & \\
Positive & & \\
\hline
\end{tabular}

A PEPI score of 0 [PT1/2, node-negative (NO), Ki67 $<2.7 \%$, estrogen receptor-positive $\left.\left(E R^{+}\right)\right]$is being investigated prospectively as a surrogate of endocrine therapy-sensitive disease that does not need chemotherapy, and a PEPI of $>0$ identifies patients with an increased risk of relapse. The hazard ratio (HR) of each surgical factor for relapse-free survival (RFS) and assigned PEPI points based on the data from the P024 trial are shown in the table. Adapted from Ma et al. (3). 
chain reaction (RT-qPCR). For each sample, the tumor area was identified and marked by the same pathologist, and this was followed by DNA extraction with the Wizard ${ }^{\complement}$ Genomic DNA Purification kit (Promega, Madison, WI, USA). DNA was quantified using Qubit Fluorometric Quantitation (Thermo Fisher Scientific, Waltham, MA, USA), and $20 \mathrm{ng} / \mu \mathrm{L}$ was the threshold for the analysis of the mutation. The reactions were performed with the equipment 7500 Fast Real-Time PCR System using TaqMan Genotyping Master Mix, primers, and TaqMan ${ }^{\circledR}$ probes and followed all recommendations of the manufacturer. A TaqMan ${ }^{(}$reference probe was used to evaluate the presence of the mutations, followed by analysis in the 7500 Software v2.06 (Thermo Fisher Scientific). The analyzed mutations were
Y537N, Y537C, Y537S, E380Q, and D538G. A description of the primer sequences used for RT-PCR can be found in Supplementary Material.

Statistical analysis was performed using Statistical Package for Social Sciences 22.0 (SPSS, Armonk, NY, USA). Categorical variables are described by frequencies and percentages. The primary endpoint is the prevalence of ESR $1 \mathrm{~m}$. For the primary endpoint, the point estimate is presented together with the exact $95 \%$ confidence interval. Continuous variables are described by means and standard deviation or median, depending on ShapiroWilk test analysis. Correlations between categorical variables were analyzed using the $\chi^{2}$ test. Differences between means are analyzed using the Student $t$-test. This project was reviewed and

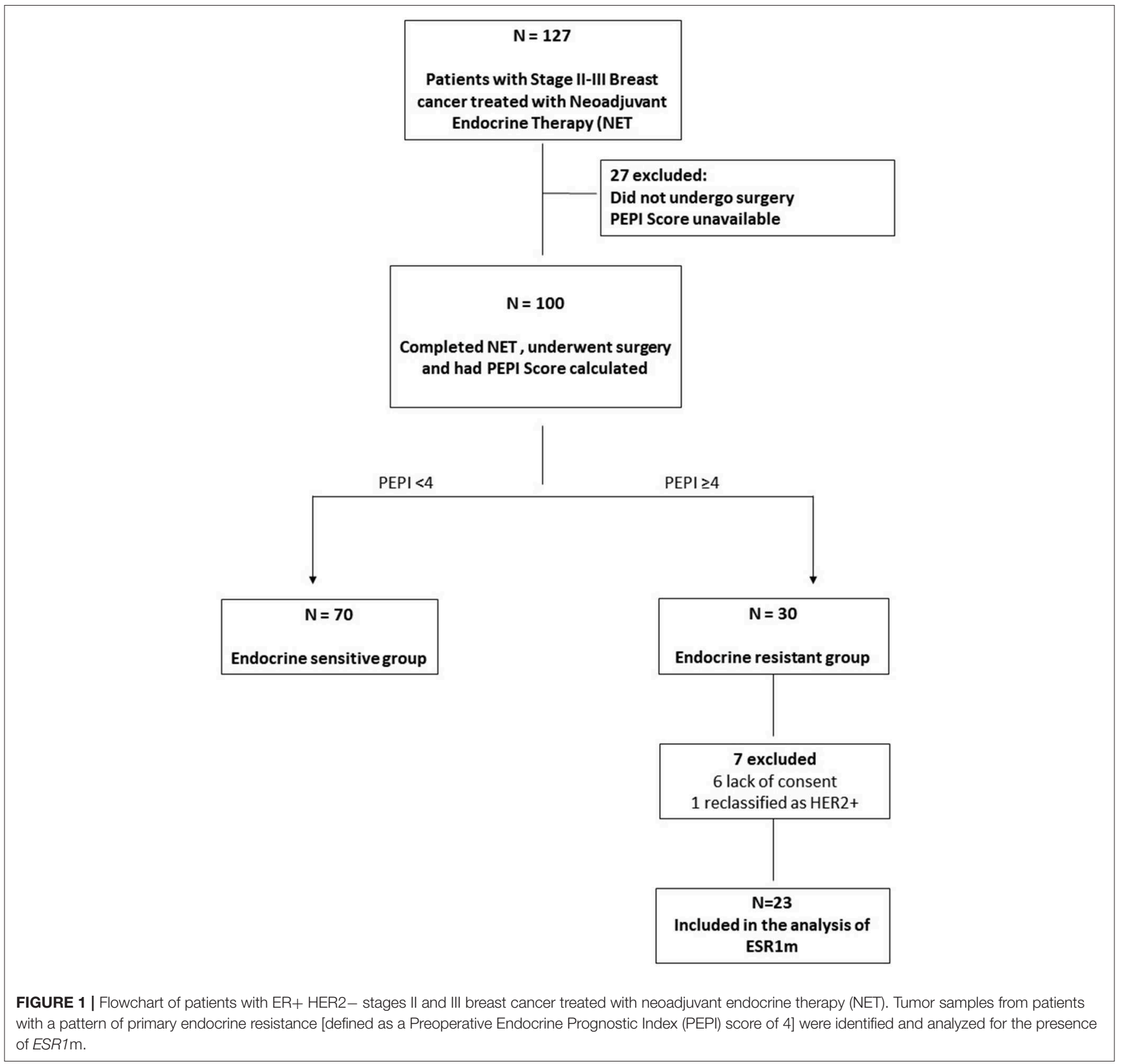


TABLE 2 | Patients characteristics, pathology and IHC results (initial biopsy sample) $(n=23)$.

\begin{tabular}{ll}
\hline Age & Median 70 years (54-84) \\
\hline Histology & Ductal: $20(86.9 \%)$ \\
& Lobular: $3(13.1 \%)$ \\
Clinical tumor size & cT1/T2: $14(60.8 \%)$ \\
& cT3/T4: $9(39.2 \%)$ \\
Clinical lymph node & Negative $(\mathrm{cN}-): 10(43.4 \%)$ \\
status & Positive (cN+): $13(56.5 \%)$ \\
Ki67 level-baseline & $<2.7 \%: 2(8.6 \%)$ \\
& $2.8-7.3 \%: 1(4.3 \%)$ \\
& $7.4-19.7 \%: 12(52.1 \%)$ \\
& $19.8-53.1 \%: 8(34.7 \%)$ \\
Duration of NET & $>53.2 \%: 0$ \\
& Median 22 weeks $(4-35)$
\end{tabular}

approved by the institutional review board (ethical committee) of both institutions.

\section{RESULTS}

One hundred twenty-seven patients were included in the cohort, of which 100 (79\%) completed NET and surgery. Among these patients, the PEPI score ranged from 0 to 3 in $70 \%$ (70/100), and $30 \%(30 / 100)$ had a PEPI score of 4 or more and were selected. Twenty-three patients were included in the analysis (six did not consent or were lost to follow-up, and one was found to be HER2positive in the surgical sample). These data are summarized in Figure 1.

Table 2 summarizes the most important characteristics of the patients and biopsy findings at the initial diagnosis. Tumors classified as primarily resistant to ET (PEPI score of four or more) were selected and had their tumors analyzed for ESR1m. In our analysis, the median age was 70 years. There was a predominance of ductal carcinoma (86.9\%) and T1-T2 tumors (60.8\%), and the majority of our patients had clinical positive axillary nodes (56.5\%) before treatment and had a Ki67 index between 7.4 and $19.7 \%$. Table 3 describes the results in terms of PEPI score and pathological and IHC evaluation. On pathological analysis (after NET), most of the tumors were T1/T2 (73.9\%), had a compromised axilla (91.3\%), maintained ER positivity (95.6\%), and had intermediate levels of Ki67.

The median duration of NET was 22 weeks (range, 4-35 weeks). All samples of tumor tissue from the surgical specimens after NET were evaluable. Quantification of DNA extraction and reference gene cycle threshold values confirmed that the material was adequate for the analysis. ESR 1 mutations were not identified in any of the 23 samples. Therefore, the prevalence of ESR $1 \mathrm{~m}$ in patients with early-stage ER+ HER2-negative breast cancer that were intrinsically resistant to NET is $0 \%$ (95\% confidence interval, $0-12.2 \%)$.

\section{DISCUSSION}

Our study reports that differently than in AI-resistant metastatic breast cancer, ESR $1 \mathrm{~m}$ are not a common mechanism of resistance
TABLE 3 | PEPI Score, pathology and IHC results after NET (surgical sample).

\begin{tabular}{ll}
\hline PEPI score & $4: 16(69.5 \%)$ \\
& $5: 2(8.6 \%)$ \\
& $6: 3(13 \%)$ \\
& $7: 2(8.6 \%)$ \\
& ypT1/T2: $17(73.9 \%)$ \\
Pathological tumor size-surgical specimen & Negative (ypN-): $2(8.6 \%)$ \\
& Positive (ypN+): \\
Pathological lymph node status & $21(91.3 \%)$ \\
& $0-2: 1(4.3 \%)$ \\
ER status, Allred score & $3-8: 22(95.6 \%)$ \\
& $<2.7 \%: 5(21.7 \%)$ \\
Ki67 level-surgical specimen & $2.8-7.3 \%: 5(21.7 \%)$ \\
& $7.4-19.7 \%: 11(47.8 \%)$ \\
& $19.8-53.1 \%: 1(4.3 \%)$ \\
& $>53.2 \%: 1(4.3 \%)$
\end{tabular}

in tumors with primary endocrine resistance in the neoadjuvant setting. This finding may have implications for the future development of ESR1m as a biomarker and therapeutic target.

Estrogen receptor-positive tumors are the most common form of breast cancer and the major cause of mortality by this disease worldwide (14). The use of endocrine agents that interfere with the ER pathway such as tamoxifen and AIs remains the standard-of-care treatment both for patients with earlystage disease and for advanced disease when the use of ET is associated with objective response and disease control in a significant proportion of patients. Nevertheless, tumor evolution caused by a complex network of mechanisms of endocrine resistance continues to be an important challenge (14). In earlystage disease, a considerable proportion of patients will develop disease recurrence despite the use of curative-intent treatment with a combination of therapies such as surgery, chemotherapy, radiation, and adjuvant hormone therapy.

Estrogen receptor, a protein encoded by the ESR1 gene, is expressed in the majority of breast cancers and is one of the key factors for disease classification and treatment definition. A significant body of research has confirmed the essential role of the ER pathway in physiological mammary gland development and also in tumorigenesis (15). Estrogen binding triggers several events, resulting in conformational changes in the ligand-binding domain (LBD), receptor activation, and allowing the ligandreceptor complex to bind to specific sequences of the genome and to interact with corepressor and coactivator proteins to regulate the transcription of estrogen-response elements (16). The most common molecular alterations in the ESR1 gene found in breast tumors are mutations, most commonly missense mutations found in codons 380, 537, and 538. ESR $1 \mathrm{~m}$ are most commonly missense mutations clustered in codons 537 and 538 of the LBD (17). The most prevalent ESR1 point mutations are Y537S and D538G, whereas others have been described at significantly lower frequencies (15). Additionally, ESR1 is known to undergo amplifications and translocations that are potential mechanisms of resistance to ET (17-19).

ESR 1m have been consistently associated with more aggressive disease, presence of visceral metastasis, and worst prognosis 
$(8,15,20)$. Extensive research addressing the potential role of ESR $1 \mathrm{~m}$ as a predictive biomarker to guide therapeutic decisions is ongoing (20). Additionally, the development of targeted therapies directed to tumor cells harboring ESR1 mutations is a logical and appealing concept, and extensive preclinical research has been conducted in this field $(4,21)$, and promising work evaluating treatment with new generation endocrine agents is in progress $(22,23)$.

The role of ESR $1 \mathrm{~m}$ has been mostly studied in the metastatic setting in patients who had disease progression on first-line ET, usually presenting with a clinical pattern of acquired (secondary) ET resistance. Nevertheless, the potential role of ESR 1m in patients with early-stage breast cancer treated with (neo)adjuvant ET has not been adequately studied. While ESR1m have been identified in advanced breast cancer, their presence in primary tumors is very low (24). Understanding mechanisms of intrinsic (acquired) endocrine resistance is of great importance, given that it could lead to the development of biomarkers and therapeutic agents that could be incorporated in the context of curativeintent treatment of breast cancer.

Historically, NET has been indicated as a clinical tool for tumor downstaging to allow breast-conserving surgery. Recently, NET is being used not only as a clinical tool but also as a scientific tool for the study of tumoral patterns of intrinsic endocrine sensitivity or resistance. Importantly, NET allows an in vivo observation of the response to estrogen deprivation therapies (25).

Preoperative Endocrine Prognostic Index, described in Figure 1, was created to distinguish tumors that are sensitive to NET from tumors that have an intrinsic degree of endocrine resistance (25). This tool is composed of four independent prognostic factors: pathological tumor size, lymph node status, level of ER expression, and Ki67 expression at the surgical sample following neoadjuvant AI therapy. The PEPI score was validated in samples from patients included in two NET clinical trials: the PO24 and IMPACT studies (11). In both trials, the rate of early relapse was very low in patients with a PEPI score of zero (25). The rate of PEPI-0 tumors in neoadjuvant AI therapy trials ranges from 17 to $37 \%$ (25). This population has a very favorable prognosis, and these patients could potentially receive adjuvant endocrine monotherapy and be spared from chemotherapy (3). Preoperative Endocrine Prognostic Index scores greater than zero were associated with an incremental increase in the risk of relapse, especially in patients with a score higher than 3 (12).

These findings were compared with a study from our group using the same methodology in patients with advanced disease where ESR 1 mutations were identified in $25 \%(n=32)$ of cases with visceral metastasis of ER+ breast cancer resistant to ET (8). A statistically significant association of the presence of ESR $1 \mathrm{~m}$ in metastatic disease compared with tumors resistant to AIs used in the neoadjuvant setting was demonstrated $(p=0.01$, Fisher exact test).

Therefore, the discovery of biomarkers associated with resistance to NET, as well as adjuvant ET, remains an unmet need. Our data reinforce the notion that mutations in the ESR1 gene do not seem to evolve rapidly and are probably mechanisms of secondary resistance to ET. Ongoing studies are evaluating a variety of potential mechanisms of primary endocrine resistance, such as defects in the DNA repair machinery (26) and aberrant FGFR signaling (27).

Our study has limitations, and we understand that the detected prevalence of ESR1m can be underestimated given the fact that a PCR-based methodology analyzed only specific mutations in the most commonly mutated codons. Therefore, it is possible that cases with mutations in different codons of the ESR1 gene potentially detectable with next-generation sequencing (NGS) technologies were not identified $(28,29)$. However, it is essential to emphasize that a robust body of literature clearly shows that ESR1m usually occurs in hotspots within the LBD, and the vast majority of the published data in this field reported mutations in the same codons we studied (15). Moreover, other studies showed that a specific real-time PCR assay does provide a rapid and reliable diagnostic tool for accurate detection of ESR1m (30).

We acknowledge that unidimensional biomarker analysis is not the best approach to investigate primary resistance and that NGS-based evaluation of whole-genome sequences, whole-exome sequencing, and even targeted gene panels could expand the identification of mechanisms of endocrine resistance. Nevertheless, the evaluation of the role of other mutations associated with endocrine resistance is beyond the scope of this article.

Additionally, and despite the challenge of conducting a prospective cohort of NET that includes more than 100 breast cancer patients, we acknowledge that the relatively low sample size of 23 cases of tumors with a PEPI score of 4 or more is a limitation and highlights the challenges of studying primary endocrine resistance. Furthermore, our patients were treated with a median 3 months of NET. Therefore, we cannot exclude that resistance documented after longer treatment durations (612 months) could not be related to receptor mutations. Still, in these cases, it would be expected that patients would have at least stable disease or a degree of response to allow for the continuation of treatment. These considerations would make it unlikely to relate ESR $1 \mathrm{~m}$ with primary resistance.

Among the strengths of our study, we highlight the conduction of a well-designed prospective cohort including 127 breast cancer patients who were treated with standardof-care NET and whose tumors were evaluated with uniform procedures of pathology and IHC. Additionally, we used a validated methodology for ESR1 determination, and we were able to perform a prespecified comparison in two different settings (neoadjuvant and metastatic). Despite the relatively low sample size, we demonstrated that the prevalence of ESR1m in NETresistant tumors is very low ( $95 \%$ of chance of being inferior to $12 \%)$. Therefore, the chances that this molecular alteration will have a practical role for patient care in this setting are minimal.

Integrative approaches to evaluate the genetic and phenotypic heterogeneity of breast tumors with the use of a more comprehensive analysis of the genome, transcriptome, epigenetic regulators, and modern quantitative proteomics methods associated with advanced bioinformatics and statistical analysis may lead to advances that can potentially be translated into improved outcomes for cancer patients (31). Advances in the understanding of the molecular biology of ER+ breast cancer led to a revolution in this field with the development of a variety 
of therapeutic agents that are now being used in routine clinical care in patients with metastatic disease such as CDK4/6, PI3K, and mTOR inhibitors (1). However, a clinically useful biomarker to identify primary sensitivity and resistance to ET has not yet been developed and remains an unmet need.

\section{CONCLUSION}

Growing evidence supports the notion that there are different mechanisms of primary and secondary endocrine resistance. Our study suggests that ESR1 mutations do not evolve rapidly and do not represent a common mechanism of primary endocrine resistance in the neoadjuvant setting. Therefore, ESR 1m should be considered a mechanism of acquired endocrine resistance in the context of advanced breast cancer. Further research should be conducted to identify factors associated with intrinsic resistance to ET.

\section{DATA AVAILABILITY STATEMENT}

All datasets generated for this study are included in the article/Supplementary Material.

\section{ETHICS STATEMENT}

All procedures performed in studies involving human participants were in accordance with the ethical standards

\section{REFERENCES}

1. Cardoso F, Senkus E, Costa A, Papadopoulos E, Aapro M, André F, et al. 4th ESO-ESMO international consensus guidelines for advanced breast cancer (ABC 4) $^{\dagger}$. Ann Oncol. (2018) 29:1634-57. doi: 10.1093/annonc/mdy192

2. Osborne CK, Schiff R. Mechanisms of endocrine resistance in breast cancer. Annu Rev Med. (2011) 62:233-47. doi: 10.1146/annurev-med-070909-182917

3. Ma CX, Reinert T, Chmielewska I, Ellis MJ. Mechanisms of aromatase inhibitor resistance. Nat Rev Cancer. (2015) 15:261-75. doi: 10.1038/nrc3920

4. Jeselsohn R, Yelensky R, Buchwalter G, Frampton G, MericBernstam F, Gonzalez-Angulo AM, et al. Emergence of constitutively active estrogen receptor- $\alpha$ mutations in pretreated advanced estrogen receptor-positive breast cancer. Clin Cancer Res. (2014) 20:1757-67. doi: 10.1158/1078-0432.CCR-13-2332

5. Kumar S, Lindsay D, Chen QB, Garrett AL, Tan XM, Anders CK, et al. Tracking plasma DNA mutation dynamics in estrogen receptor positive metastatic breast cancer with dPCR-SEQ. NPJ Breast Cancer. (2018) 4:39. doi: 10.1038/s41523-018-0093-3

6. Chu D, Paoletti C, Gersch C, VanDenBerg DA, Zabransky DJ, Cochran RL, et al. ESR1 mutations in circulating plasma tumor DNA from metastatic breast cancer patients. Clin Cancer Res. (2016) 22:993-9. doi: 10.1158/1078-0432.CCR15-0943

7. Schiavon G, Hrebien S, Garcia-Murillas I, Cutts RJ, Pearson A, Tarazona N, et al. Analysis of ESR1 mutation in circulating tumor DNA demonstrates evolution during therapy for metastatic breast cancer. Sci Transl Med. (2015) 7:313ra182. doi: 10.1126/scitranslmed.aac7551

8. Reinert T, Coelho GP, Mandelli J, Zimermann E, Zaffaroni F, Bines J, et al. Association of ESR1 mutations and visceral metastasis in patients with estrogen receptor-positive advanced breast cancer from Brazil. J Oncol. (2019) 2019:1947215. doi: 10.1155/2019/1947215 of the institutional and/or national research committee and with the 1964 Helsinki declaration and its later amendments or comparable ethical standards. Informed consent was obtained from all individual participants included in the study. This article does not contain any studies with animals performed by any of the authors.

\section{AUTHOR CONTRIBUTIONS}

TR, SR, CC, JB, CB, ME, and MG designed the study. TR, SR, VV, LS, CC, CA, AS, and MK were involved in the oncologic treatment of patients. GC, JM, GP, and MG did the pathologic and molecular biology evaluation. TR, SR, VV, and MB analyzed and interpreted the data. All authors read and approved the final manuscript.

\section{FUNDING}

This work was supported by AstraZeneca Brazil through an investigator-initiated grant (ESR 17-12682).

\section{SUPPLEMENTARY MATERIAL}

The Supplementary Material for this article can be found online at: https://www.frontiersin.org/articles/10.3389/fonc. 2020.00342/full\#supplementary-material

9. Jeselsohn R, Buchwalter G, De Angelis C, Brown M, Schiff R. ESR1 mutations - a mechanism for acquired endocrine resistance in breast cancer. Nat Rev Clin Oncol. (2015) 12:573-83. doi: 10.1038/nrclinonc.2015.117

10. Ramalho S, Ramalh S, Gonçalves R, Barrios CH, Graudenz MS, Bines J. Multidisciplinary approach to neoadjuvant endocrine therapy in breast cancer: a comprehensive review. Rev Bras Ginecol Obstet. (2016) 38:61522. doi: 10.1055/s-0036-1597579

11. Reinert T, Gonçalves R, Ellis MJ. Current status of neoadjuvant endocrine therapy in early stage breast cancer. Curr Treat Options Oncol. (2018) 19:23. doi: 10.1007/s11864-018-0538-9

12. Ellis MJ, Suman VJ, Hoog J, Goncalves R, Sanati S, Creighton CJ, et al. Ki67 proliferation index as a tool for chemotherapy decisions during and after neoadjuvant aromatase inhibitor treatment of breast cancer: results from the American college of surgeons oncology group Z1031 trial (alliance). J Clin Oncol. (2017) 35:1061-9. doi: 10.1200/JCO.2016.69.4406

13. Amoroso V, Generali D, Buchholz T, Cristofanilli M, Pedersini R, Curigliano $\mathrm{G}$, et al. International expert consensus on primary systemic therapy in the management of early breast cancer: highlights of the fifth symposium on primary systemic therapy in the management of operable breast cancer, cremona, Italy (2013). J Natl Cancer Inst Monogr. (2015) 2015: 906. doi: 10.1093/jncimonographs/lgv023

14. Tryfonidis K, Zardavas D, Katzenellenbogen BS, Piccart M. Endocrine treatment in breast cancer: cure, resistance and beyond. Cancer Treat Rev. (2016) 50:68-81. doi: 10.1016/j.ctrv.2016.08.008

15. Reinert T, Saad ED, Barrios CH, Bines J. Clinical implications of ESR1 mutations in hormone receptor-positive advanced breast cancer. Front Oncol. (2017) 7:26. doi: 10.3389/fonc.2017.00026

16. Schiff R, Massarweh S, Shou J, Osborne CK. Breast cancer endocrine resistance: how growth factor signaling and estrogen receptor coregulators modulate response. Clin Cancer Res. (2003) 9(1 Pt 2):447S-54S. doi: 10.1158/1078-0432.ccr-031212 
17. Fanning SW, Mayne CG, Dharmarajan V, Carlos KE, Martin TA, Novick SJ, et al. Estrogen receptor alpha somatic mutations Y537S and D538G confer breast cancer endocrine resistance by stabilizing the activating function-2 binding conformation. Breast Cancer Treat. (2017) 9: 4628. doi: 10.7554/eLife.12792

18. Li S, Shen D, Shao J, Crowder R, Liu W, Prat A, et al. Endocrine-therapyresistant ESR1 variants revealed by genomic characterization of breast-cancerderived xenografts. Cell Rep. (2013) 4:1116-30. doi: 10.1016/j.celrep.2013. 08.022

19. Holst F. Estrogen receptor alpha gene amplification in breast cancer: 25 years of debate. World J Clin Oncol. (2016) 7:160-73. doi: 10.5306/wjco.v7.i2.160

20. Jeselsohn R, De Angelis C, Brown M, Schiff R. The evolving role of the estrogen receptor mutations in endocrine therapy-resistant breast cancer. Curr Oncol Rep. (2017) 19:35. doi: 10.1007/s11912-0170591-8

21. Spoerke JM, Gendreau S, Walter K, Qiu J, Wilson TR, Savage H, et al. Heterogeneity and clinical significance of ESR1 mutations in ER-positive metastatic breast cancer patients receiving fulvestrant. Nat Commun. (2016) 7:11579. doi: 10.1038/ncomms 11579

22. Weir HM, Bradbury RH, Lawson M, Rabow AA, Buttar D, Callis RJ, et al. AZD9496: an oral estrogen receptor inhibitor that blocks the growth of ER-positive and ESR1-mutant breast tumors in preclinical models. Cancer Res. (2016) 76:3307-18. doi: 10.1158/0008-5472.CAN-1 5-2357

23. Fanning SW, Jeselsohn R, Dharmarajan V, Mayne CG, Karimi M, Buchwalter G, et al. The SERM/SERD bazedoxifene disrupts ESR1 helix 12 to overcome acquired hormone resistance in breast cancer cells. Elife. (2018) 7:e37161. doi: 10.7554/eLife. 37161

24. Bertucci, F, Ng CKY, Patsouris A, Droin N, Piscuoglio S, Carbuccia A, et al. Genomic characterization of metastatic breast cancer. Nature. (2019) 569:560-4. doi: 10.1038/s41586-0191056-Z

25. Ellis MJ, Tao Y, Luo J, A'Hern R, Evans DB, Bhatnagar AS, et al. Outcome prediction for estrogen receptor-positive breast cancer based on postneoadjuvant endocrine therapy tumor characteristics. J Natl Cancer Inst. (2008) 100:1380-8. doi: 10.1093/jnci/djn309
26. Haricharan S, Ellis MJ. Defects in mismatch repair: the achilles heel of estrogen receptor positive breast cancer with intrinsic endocrine therapy resistance? Oncoscience. (2017) 4:77-8. doi: 10.18632/oncoscience.363

27. Formisano L, Lu Y, Servetto A, Hanker AB, Jansen VM, Bauer JA, et al. Aberrant FGFR signaling mediates resistance to CDK4/6 inhibitors in ER+ breast cancer. Nat Commun. (2019) 10:1373. doi: 10.1038/s41467-019-09068-2

28. Shaw JA, Guttery DS, Hills A, Fernandez-Garcia D, Page K, Rosales BM, et al. Mutation analysis of cell-free DNA and single circulating tumor cells in metastatic breast cancer patients with high circulating tumor cell counts. Clin Cancer Res. (2017) 23:88-96. doi: 10.1158/1078-0432.CCR-16-0825

29. Jeselsohn R. Are we ready to use ESR1 mutations in clinical practice? Breast Care. (2017) 12:309-13. doi: 10.1159/000481428

30. Wang T, Liu JH, Zhang J, Wang L, Chen C, Dai PG, et al. A multiplex allele-specific real-time PCR assay for screening of ESR1 mutations in metastatic breast cancer. Exp Mol Pathol. (2015) 98:1527. doi: 10.1016/j.yexmp.2015.03.004

31. Cieslik M, Chinnaiyan AM. Cancer transcriptome profiling at the juncture of clinical translation. Nat Rev Genet. (2018) 19:93-109. doi: 10.1038/nrg.2017.96

Conflict of Interest: TR received research funding from AstraZeneca and speaker honoraria from AstraZeneca, Lilly, Novartis, Pfizer, and PierreFabre. CB received research funding, speaker honoraria and participated in advisory boards from Roche, Pfizer, GSK, Boehringer Ingelhein, Eisai, AstraZeneca, BMS, MSD, Libbs.

The remaining authors declare that the research was conducted in the absence of any commercial or financial relationships that could be construed as a potential conflict of interest.

Copyright (c) 2020 Reinert, Ramalho, de Vasconcelos, Silva, da Silva, de Andrade, Kraft, Coelho, Mandelli, Binotto, Cabello, Paiva Silva, Bines, Barrios, Ellis and Graudenz. This is an open-access article distributed under the terms of the Creative Commons Attribution License (CC BY). The use, distribution or reproduction in other forums is permitted, provided the original author(s) and the copyright owner(s) are credited and that the original publication in this journal is cited, in accordance with accepted academic practice. No use, distribution or reproduction is permitted which does not comply with these terms. 